\title{
STABILITY OF THE BLASCHKE-SANTALÓ INEQUALITY IN THE PLANE
}

\author{
MOHAMMAD N. IVAKI
}

\begin{abstract}
We give a stability version of of the Blaschke-Santaló inequality in the plane.
\end{abstract}

\section{INTRODUCTION}

The setting of this paper is the $n$-dimensional Euclidean space. A compact convex subset of $\mathbb{R}^{n}$ with non-empty interior is called a convex body. The set of convex bodies in $\mathbb{R}^{n}$ is denoted by $\mathcal{K}^{n}$. Write $\mathcal{K}_{e}^{n}$ for the set of origin-symmetric convex bodies and $\mathcal{K}_{0}^{n}$ for the set of convex bodies whose interiors contain the origin.

The support function of $K \in \mathcal{K}^{n}, h_{K}: \mathbb{S}^{n-1} \rightarrow \mathbb{R}$, is defined by

$$
h_{K}(u)=\max _{x \in K}\langle x, u\rangle,
$$

where $\langle\cdot, \cdot\rangle$ stands for the usual inner product of $\mathbb{R}^{n}$. The polar body, $K^{*}$, of $K \in \mathcal{K}_{0}^{n}$ is the convex body defined by

$$
K^{*}=\left\{y \in \mathbb{R}^{n}:\langle x, y\rangle \leq 1 \text { for all } x \in K\right\} .
$$

For $x \in \operatorname{int} K$, let $K^{x}:=(K-x)^{*}$. The Santaló point of $K$, denoted by $s$, is the unique point in int $K$ such that

$$
V\left(K^{s}\right) \leq V\left(K^{x}\right)
$$

for all $x \in \operatorname{int} K$. For a body $K \in \mathcal{K}_{e}^{n}$, the Santaló point is at the origin. The Blaschke-Santaló inequality $[4,21]$ states that

$$
V\left(K^{s}\right) V(K) \leq \omega_{n}^{2}
$$

with equality if and only if $K$ is an ellipsoid. Here $\omega_{n}$ is the volume of $B$, the unit ball of $\mathbb{R}^{n}$. The equality condition was settled by Saint Raymond [20] in the symmetric case and Petty [19] in the general case.

A natural tool in the affine geometry of convex bodies is the Banach-Mazur distance which for two convex bodies $K, \bar{K} \in \mathcal{K}^{n}$ is defined by

$d_{\mathcal{B} \mathcal{M}}(K, \bar{K})=\min \left\{\lambda \geq 1:(K-x) \subseteq \Phi(\bar{K}-y) \subseteq \lambda(K-x), \Phi \in G L(n), x, y \in \mathbb{R}^{n}\right\}$. It is easy to see that $d_{\mathcal{B M}}(K, \Phi \bar{K})=d_{\mathcal{B M}}(K, \bar{K})$ for all $\Phi \in G L(n)$. Moreover, the Banach-Mazur distance is multiplicative. That is, for $K_{1}, K_{2}, K_{3} \in \mathcal{K}_{e}^{n}$ the following inequality holds:

$$
d_{\mathcal{B M}}\left(K_{1}, K_{3}\right) \leq d_{\mathcal{B M}}\left(K_{1}, K_{2}\right) d_{\mathcal{B M}}\left(K_{2}, K_{3}\right) .
$$

The main result of the paper is stated in the following theorem.

2010 Mathematics Subject Classification. Primary 52A40, 52A10; Secondary 53A15.

Key words and phrases. the Blaschke-Santaló inequality; stability of the Blaschke-Santaló inequality. 
Theorem. There exist constants $\gamma, \varepsilon_{0}>0$, such that the following holds: If $0<\varepsilon<\varepsilon_{0}$ and $K$ is a convex body in $\mathbb{R}^{2}$ such that $V\left(K^{s}\right) V(K) \geq \frac{\pi^{2}}{1+\varepsilon}$, then $d_{\mathcal{B M}}(K, B) \leq 1+\gamma \varepsilon^{\frac{1}{4}}$. Furthermore, if $K$ is an origin-symmetric body, then $d_{\mathcal{B M}}(K, B) \leq 1+\gamma \varepsilon^{\frac{1}{2}}$.

In $\mathbb{R}^{n}, n \geq 3$, the stability of the Blaschke-Santaló inequality was first proved by K.J. Böröczky [6], and then by K. Ball and K.J. Böröczky [2] with a better order of approximation (see also [3] for the stability of functional forms of the BlaschkeSantaló inequality). In $\mathbb{R}^{2}$, a result has been obtained by K.J. Böröczky and E. Makai [7] where the order of approximation in the origin-symmetric case is $1 / 3$ and in the general case is $1 / 6$. Therefore, our main theorem provides a sharper stability result. Moreover, stability of the $p$-affine isoperimetric inequality also follows from the stability of the Blaschke-Santaló inequality (See [17, 22] for definitions of the $p$-affine surface areas, and for the statements of the $p$-affine isoperimetric inequalities, and see also $[13,14]$ for their generalizations in the context of the Orlicz-Brunn-Minkowski theory, basic properties, and affine isoperimetric inequalities they satisfy.). Stability of the $p$-affine isoperimetric inequality, in the Hausdorff distance, for bodies in $\mathcal{K}_{e}^{2}$ was established by the author in [12] via the affine normal flow with the order of approximation equal to $3 / 10$. Therefore, the main theorem here replaces $3 / 10$ by $1 / 2$ and extends that result, if $p>1$, to bodies with the Santaló points or centroids at the origin, and if $p=1$, to any convex body in $\mathcal{K}^{2}$. An application of such a stability result to some Monge-Ampère functionals is given by Ghilli and Salani [9].

Acknowledgment. I am indebted to Monika Ludwig and the referee for the very careful reading of the original submission.

\section{BACKGROUND MATERIAL}

A convex body is said to be of class $\mathcal{C}_{+}^{k}$, for some $k \geq 2$, if its boundary hypersurface is $k$-times continuously differentiable, in the sense of differential geometry, and the Gauss map $\nu: \partial K \rightarrow \mathbb{S}^{n-1}$, which takes $x$ on the boundary of $K$ to its unique outer unit normal vector $\nu(x)$, is well-defined and a $\mathcal{C}^{k-1}$-diffeomorphism.

Let $K, L$ be two convex bodies and $0<a<\infty$, then the Minkowski sum $K+a L$ is defined by $h_{K+a L}=h_{K}+a h_{L}$ and the mixed volume $V_{1}(K, L)(V(K, L)$ for planar convex bodies) of $K$ and $L$ is defined by

$$
V_{1}(K, L)=\frac{1}{n} \lim _{a \rightarrow 0^{+}} \frac{V(K+a L)-V(K)}{a} .
$$

A fundamental fact is that corresponding to each convex body $K$, there is a unique Borel measure $S_{K}$ on the unit sphere such that

$$
V_{1}(K, L)=\frac{1}{n} \int_{\mathbb{S}^{n-1}} h_{L} d S_{K}
$$

for any convex body $L$. The measure $S_{K}$ is called the surface area measure of $K$.

A convex body $K$ is said to have a positive continuous curvature function $f_{K}$, defined on the unit sphere, provided that for each convex body $L$

$$
V_{1}(K, L)=\frac{1}{n} \int_{\mathbb{S}^{n-1}} h_{L} f_{K} d \sigma
$$


where $\sigma$ is the spherical Lebesgue measure on $\mathbb{S}^{n-1}$. A convex body can have at most one curvature function; see [5, p. 115]. If $K$ is of class $\mathcal{C}_{+}^{2}$, then $S_{K}$ is absolutely continuous with respect to $\sigma$, and the Radon-Nikodym derivative $d S_{K} / d \sigma: \mathbb{S}^{n-1} \rightarrow$ $\mathbb{R}$ is the reciprocal Gauss curvature of $\partial K$ (viewed as a function of the outer unit normal vectors). For every $K \in \mathcal{K}^{n}, V(K)=V_{1}(K, K)$.

Of significant importance in convex geometry is the Minkowski mixed volume inequality. Minkowski's mixed volume inequality states that for $K, L \in \mathcal{K}^{n}$,

$$
V_{1}(K, L)^{n} \geq V(K)^{n-1} V(L) .
$$

In the class of origin-symmetric convex bodies, equality holds if and only if $K=c L$ for some $c>0$. In $\mathbb{R}^{2}$ a stronger version of Minkowski's inequality was obtained by Groemer [10]. We provide his result for bodies in $\mathcal{K}_{e}^{2}$ :

Theorem 1. [10] Let $K, L \in \mathcal{K}_{e}^{2}$ and set $D(K)=2 \max _{\mathbb{S}^{1}} h_{K}$, then

$$
\frac{V(K, L)^{2}}{V(K) V(L)}-1 \geq \frac{V(K)}{4 D^{2}(K)} \max _{u \in \mathbb{S}^{1}}\left|\frac{h_{K}(u)}{V(K)^{\frac{1}{2}}}-\frac{h_{L}(u)}{V(L)^{\frac{1}{2}}}\right|^{2} .
$$

The Santaló point of $K$ is characterized by the following property

$$
\int_{\mathbb{S}^{n-1}} \frac{u}{h_{K-s}^{n+1}(u)} d \sigma(u)=0 .
$$

Thus for an arbitrary convex body $K$, the indefinite $\sigma$-integral of $h_{K-s}^{-(n+1)}$ satisfies the sufficiency condition of Minkowski's existence theorem in $\mathbb{R}^{n}$ (see, for example, Schneider [22, Theorem 8.2.2]). Hence, there exists a unique convex body (up to translation) with curvature function

$$
f_{\Lambda K}=\frac{V(K)}{V\left(K^{s}\right)} h_{K-s}^{-(n+1)} .
$$

Moreover, $\Lambda \Phi K=\Phi \Lambda K$ (up to translation) for $\Phi \in G L(n)$, by [16, Lemma 7.12]. Finally, we remark that by the Minkowski inequality for all $L \in \mathcal{K}^{2}$ there holds $V^{2}(L)=V(\Lambda L, L)^{2} \geq V(L) V(\Lambda L)$. Therefore $V(L) \geq V(\Lambda L)$ for all $L \in \mathcal{K}^{2}$, with equality if and only if $\Lambda L$ is a translate of $L$. In this paper we always assume that the centroid of $\Lambda K$ is the origin of the plane.

Remark 2. If $K \in \mathcal{K}^{n}$ is of class $\mathcal{C}_{+}^{\infty}$, then $h_{K} \in \mathcal{C}^{\infty}$. In fact, by definition of the $\operatorname{class} \mathcal{C}_{+}^{\infty}$, the Gauss map $\nu$ is a diffeomorphism of class $\mathcal{C}^{\infty}$ and so $h_{K}(\cdot)=\left\langle\nu^{-1}(\cdot), \cdot\right\rangle$ is of class $\mathcal{C}^{\infty}$. In this case, since $\Lambda K$ is a solution to the Minkowski problem (2.2) with positive $\mathcal{C}^{\infty}$ prescribed data $\frac{V(K)}{V\left(K^{s}\right)} h_{K-s}^{-(n+1)}, \Lambda K$ is of class $\mathcal{C}_{+}^{\infty}$; see Cheng and Yau [8, Theorem 1].

Theorem 3. [11] Suppose that $K \in \mathcal{K}_{e}^{2}$ is of class $\mathcal{C}_{+}^{\infty}$. If $m \leq h_{K} f_{K}^{1 / 3} \leq M$ for some positive numbers $m$ and $M$, then there exist two ellipses $E_{\text {in }}$ and $E_{\text {out }}$ such that $E_{\text {in }} \subseteq K \subseteq E_{\text {out }}$ and

$$
\left(\frac{V\left(E_{\text {in }}\right)}{\pi}\right)^{2 / 3}=m,\left(\frac{V\left(E_{\text {out }}\right)}{\pi}\right)^{2 / 3}=M
$$

Corollary 4. Suppose that $K \in \mathcal{K}_{e}^{2}$ is of class $\mathcal{C}_{+}^{\infty}$. If $m \leq h_{K} f_{K}^{1 / 3} \leq M$ for some positive numbers $m$ and $M$ and $V(K)=\pi$, then $m \leq 1 \leq M$. Moreover, without 
any assumption on the area of $K$, we have

$$
d_{\mathcal{B M}}(K, B) \leq\left(\frac{M}{m}\right)^{\frac{3}{2}} .
$$

Proof. Let $E_{\text {in }}$ and $E_{\text {out }}$ be the ellipses from Theorem 3. Since $V\left(E_{\text {out }}\right) \geq \pi$ and $V\left(E_{i n}\right) \leq \pi$, the first claim follows (For another proof by Andrews, see [1, Lemma 10] in which he does not assume that $K$ is origin-symmetric.). To prove the bound on the Banach-Mazur distance, we may first apply a special linear transformation $\Phi \in S L(2)$ such that $\Phi E_{\text {out }}$ is a disk. Then it is easy to see that $\Phi E_{\text {out }} \subseteq \frac{V\left(E_{\text {out }}\right)}{V\left(E_{\text {in }}\right)} \Phi E_{\text {in }}$. Therefore

$$
\Phi E_{\text {in }} \subseteq \Phi K \subseteq \frac{V\left(E_{\text {out }}\right)}{V\left(E_{\text {in }}\right)} \Phi E_{\text {in }},
$$

and

$$
d_{\mathcal{B M}}(K, B) \leq \frac{V\left(E_{\text {out }}\right)}{V\left(E_{\text {in }}\right)}
$$

Let $K$ be a convex body with Santaló point at the origin. In [15], by using the affine isoperimetric inequality, Lutwak proved

$$
V(K) V\left(K^{*}\right) \leq \omega_{n}^{2}\left(\frac{V(\Lambda K)}{V(K)}\right)^{n-1} .
$$

We will use this inequality for $n=2$ in the proof of the main theorem.

\section{Proof of the main theorem}

We shall begin by proving the claim for bodies in $\mathcal{K}_{e}^{2}$ that are of class $\mathcal{C}_{+}^{\infty}$. By John's ellipsoid theorem, we may assume without losing any generality, after applying a $G L(2)$ transformation, that

$$
1 \leq h_{K} \leq \sqrt{2} .
$$

In view of inequality (2.3), inequality $V(K) V\left(K^{*}\right) \geq \frac{\pi^{2}}{1+\varepsilon}$ gives

$$
1 \geq \frac{V(\Lambda K)}{V(K)} \geq \frac{1}{1+\varepsilon} .
$$

We will rewrite (3.2) as the following equivalent expression

$$
\frac{V(K, \Lambda K)^{2}}{V(\Lambda K) V(K)}-1 \leq \varepsilon
$$

Therefore, by Groemer's stability theorem, (2.1), we obtain

$$
\frac{V(K)}{4 D^{2}(K)} \max _{u \in \mathbb{S}^{1}}\left|\frac{h_{K}(u)}{V(K)^{\frac{1}{2}}}-\frac{h_{\Lambda K}(u)}{V(\Lambda K)^{\frac{1}{2}}}\right|^{2} \leq \varepsilon .
$$

Thus for every $u \in \mathbb{S}^{1}$ there holds

$$
\frac{h_{K}^{2}(u)}{V(K)}\left|\frac{V(\Lambda K)^{\frac{1}{2}}}{V(K)^{\frac{1}{2}}}-\frac{h_{\Lambda K}(u)}{h_{K}(u)}\right|^{2} \leq \frac{h_{K}^{2}(u)}{V(\Lambda K)}\left|\frac{V(\Lambda K)^{\frac{1}{2}}}{V(K)^{\frac{1}{2}}}-\frac{h_{\Lambda K}(u)}{h_{K}(u)}\right|^{2} \leq \frac{32}{\pi} \varepsilon .
$$


Using (3.1) we can estimate the left-hand side of (3.3) to obtain

$$
\max _{u \in \mathbb{S}^{1}}\left|\frac{V(\Lambda K)^{\frac{1}{2}}}{V(K)^{\frac{1}{2}}}-\frac{h_{\Lambda K}(u)}{h_{K}(u)}\right|^{2} \leq 64 \varepsilon .
$$

Recall from (2.2) that

$$
h_{K}=\left(\frac{V(K)}{V\left(K^{*}\right)}\right)^{\frac{1}{3}} \frac{1}{f_{\Lambda K}^{\frac{1}{3}}} .
$$

Plugging this into (3.4) gives

$$
\left(\frac{V\left(K^{*}\right)}{V(K)}\right)^{\frac{2}{3}} \max _{u \in \mathbb{S}^{1}}\left|\frac{V(\Lambda K)^{\frac{1}{2}}}{V(K)^{\frac{1}{2}}}\left(\frac{V(K)}{V\left(K^{*}\right)}\right)^{\frac{1}{3}}-\left(h_{\Lambda K} f_{\Lambda K}^{\frac{1}{3}}\right)(u)\right|^{2} \leq 64 \varepsilon .
$$

On the other hand, as (3.1) also implies $\frac{1}{\sqrt{2}} \leq h_{K^{*}} \leq 1$, we deduce that

$$
\max _{u \in \mathbb{S}^{1}}\left|\frac{V(\Lambda K)^{\frac{1}{2}}}{V(K)^{\frac{1}{2}}}\left(\frac{V(K)}{V\left(K^{*}\right)}\right)^{\frac{1}{3}}-\left(h_{\Lambda K} f_{\Lambda K}^{\frac{1}{3}}\right)(u)\right|^{2} \leq(64) 4^{\frac{2}{3}} \varepsilon .
$$

In particular, this last inequality leads us to

$$
\max _{u \in \mathbb{S}^{1}}\left(h_{\Lambda K} f_{\Lambda K}^{\frac{1}{3}}\right)(u)-\min _{u \in \mathbb{S}^{1}}\left(h_{\Lambda K} f_{\Lambda K}^{\frac{1}{3}}\right)(u) \leq 2^{\frac{25}{6}} \varepsilon^{\frac{1}{2}} .
$$

By multiplying $\Lambda K$ with $\sqrt{\frac{\pi}{V(\Lambda K)}}$ we have $V\left(\sqrt{\frac{\pi}{V(\Lambda K)}} \Lambda K\right)=\pi$. So by Remark 2, Corollary 4, and (3.5) we get

$$
2^{\frac{25}{6}} \varepsilon^{\frac{1}{2}}\left(\frac{\pi}{V(\Lambda K)}\right)^{2 / 3}+1 \geq\left(\frac{\pi}{V(\Lambda K)}\right)^{2 / 3} \max _{\mathbb{S}^{1}}\left(h_{\Lambda K} f_{\Lambda K}^{\frac{1}{3}}\right),
$$

and

$$
1-2^{\frac{25}{6}} \varepsilon^{\frac{1}{2}}\left(\frac{\pi}{V(\Lambda K)}\right)^{2 / 3} \leq\left(\frac{\pi}{V(\Lambda K)}\right)^{2 / 3} \min _{\mathbb{S}^{1}}\left(h_{\Lambda K} f_{\Lambda K}^{\frac{1}{3}}\right) .
$$

Furthermore, notice that by (3.1) and (3.2) the following inequality holds:

$$
1-2^{\frac{25}{6}} \varepsilon^{\frac{1}{2}}\left(\frac{\pi}{V(\Lambda K)}\right)^{2 / 3} \geq 1-2^{\frac{25}{6}} \varepsilon^{\frac{1}{2}}(1+\varepsilon)^{\frac{2}{3}} .
$$

Take $\varepsilon$ small enough such that

$$
1-2^{\frac{25}{6}} \varepsilon^{\frac{1}{2}}(1+\varepsilon)^{2 / 3}>0 .
$$

So far we have proved: If $\varepsilon$ is small enough, then

$$
\max _{\mathbb{S}^{1}}\left(h_{\Lambda K} f_{\Lambda K}^{\frac{1}{3}}\right) \leq\left(1+2^{\frac{25}{6}} \varepsilon^{\frac{1}{2}}(1+\varepsilon)^{2 / 3}\right)\left(\frac{\pi}{V(\Lambda K)}\right)^{-2 / 3},
$$

and

$$
\min _{\mathbb{S}^{1}}\left(h_{\Lambda K} f_{\Lambda K}^{\frac{1}{3}}\right) \geq\left(1-2^{\frac{25}{6}} \varepsilon^{\frac{1}{2}}(1+\varepsilon)^{2 / 3}\right)\left(\frac{\pi}{V(\Lambda K)}\right)^{-2 / 3}>0 .
$$

With the aid of these last inequalities and Corollary 4 we deduce that

$$
d_{\mathcal{B} \mathcal{M}}(\Lambda K, B) \leq\left(\frac{1+2^{\frac{25}{6}} \varepsilon^{\frac{1}{2}}(1+\varepsilon)^{2 / 3}}{1-2^{\frac{25}{6}} \varepsilon^{\frac{1}{2}}(1+\varepsilon)^{2 / 3}}\right)^{3 / 2} .
$$


We return to inequality (3.4) and combine it with (3.2) to get

$$
-8 \varepsilon^{\frac{1}{2}}+\frac{1}{(1+\varepsilon)^{\frac{1}{2}}} \leq-8 \varepsilon^{\frac{1}{2}}+\frac{V(\Lambda K)^{\frac{1}{2}}}{V(K)^{\frac{1}{2}}} \leq \frac{h_{\Lambda K}}{h_{K}} \leq 8 \varepsilon^{\frac{1}{2}}+\frac{V(\Lambda K)^{\frac{1}{2}}}{V(K)^{\frac{1}{2}}} \leq 1+8 \varepsilon^{\frac{1}{2}} .
$$

Furthermore, take $\varepsilon$ small enough such that $-8 \varepsilon^{\frac{1}{2}}+\frac{1}{(1+\varepsilon)^{\frac{1}{2}}}>0$. Consequently

$$
d_{\mathcal{B M}}(K, \Lambda K) \leq \frac{1+8 \varepsilon^{\frac{1}{2}}}{-8 \varepsilon^{\frac{1}{2}}+\frac{1}{(1+\varepsilon)^{\frac{1}{2}}}} .
$$

Taking into account (3.6), (3.7), and the multiplicativity of the Banach-Mazur distance results in the desired estimate:

$$
d_{\mathcal{B M}}(K, B) \leq\left(\frac{1+2^{\frac{25}{6}} \varepsilon^{\frac{1}{2}}(1+\varepsilon)^{2 / 3}}{1-2^{\frac{25}{6}} \varepsilon^{\frac{1}{2}}(1+\varepsilon)^{2 / 3}}\right)^{3 / 2}\left(\frac{1+8 \varepsilon^{\frac{1}{2}}}{-8 \varepsilon^{\frac{1}{2}}+\frac{1}{(1+\varepsilon)^{\frac{1}{2}}}}\right) \leq 1+\gamma \varepsilon^{\frac{1}{2}},
$$

for some universal $\gamma>0$, provided that $\varepsilon$ is small enough.

It follows from [22, Section 3.4] that the class of $\mathcal{C}_{+}^{\infty}$ origin-symmetric convex bodies is dense in $\mathcal{K}_{e}^{n}$. Therefore, an approximation argument will prove that the claim of the main theorem, in fact, holds for any origin-symmetric convex body. To get the more general result, for bodies in $\mathcal{K}^{2}$, we will first need to recall Theorem 1.4 of Böröczky from [6] and a theorem of Meyer and Pajor from [18]:

Theorem (Böröczky, [6]). For any convex body $K$ in $\mathbb{R}^{n}$ with $d_{\mathcal{B M}}(K, B) \geq 1+\varepsilon$ for $\varepsilon>0$, there exists an origin-symmetric convex body $C$ and a constant $\gamma^{\prime}>0$ depending on $n$ such that $d_{\mathcal{B M}}(C, B) \geq 1+\gamma^{\prime} \varepsilon^{2}$ and $C$ results from $K$ as a limit of subsequent Steiner symmetrizations and affine transformations.

Theorem (Meyer, Pajor, [18]). Let $K$ be a convex body in $\mathbb{R}^{n}, H$ be a hyperplane, and let $K_{H}$ be the Steiner symmetral of $K$ with respect to $H$. If $s$ and $s^{\prime}$ denote the Santaló points of $K$ and $K_{H}$, respectively, then $s^{\prime} \in H$, and $V\left(K^{s}\right) \leq V\left(\left(K_{H}\right)^{s^{\prime}}\right)$.

Now we give the proof in the general case by contraposition. Let $K$ be a convex body such that

$$
d_{\mathcal{B M}}(K, B)>1+\left(\frac{\gamma}{\gamma^{\prime}}\right)^{\frac{1}{2}} \varepsilon^{\frac{1}{4}}
$$

where $\gamma^{\prime}$ is the constant in Böröczky's theorem. So by the last two theorems, there exists an origin-symmetric convex body $C$, such that $V(C) V\left(C^{*}\right) \geq V(K) V\left(K^{s}\right)$ and $d_{\mathcal{B M}}(C, B)>1+\gamma \varepsilon^{\frac{1}{2}}$. Moreover, $d_{\mathcal{B M}}(C, B)>1+\gamma \varepsilon^{\frac{1}{2}}$ implies that

$$
V(C) V\left(C^{*}\right)<\frac{\pi^{2}}{1+\varepsilon} .
$$

Therefore

$$
V(K) V\left(K^{s}\right)<\frac{\pi^{2}}{1+\varepsilon}
$$

The argument is complete. 


\section{REFERENCES}

1. B. Andrews, The affine curve-lengthening flow. J. Reine Angew. Math. 506, 43-83 (1999)

2. K. Ball, K.J. Böröczky, Stability of some versions of the Prékopa-Leindler inequality. Monatsh. Math. 163, 1-14 (2011)

3. F. Barthe, K.J. Böröczky, M. Fradelizi, Stability of the functional forms of the BlaschkeSantaló inequality. Monatsh. Math. 173, 135-159 (2014)

4. W. Blaschke, Über affine Geometrie I: Isoperimetrische Eigenschaften von Ellipse und Ellipsoid. Ber. Verh. Sächs. Akad. Leipzig, Math.-Phys. Kl. 68, 217-39 (1916)

5. T. Bonnesen, W. Fenchel, Theorie der konvexen Körper. Springer-Verlag, Berlin, (1934)

6. K.J. Böröczky, Stability of Blaschke-Santaló inequality and the affine isoperimetric inequality. Adv. in Math. 225, 1914-1928 (2010)

7. K.J. Böröczky, E. Makai, Jr. On the volume product of planar polar convex bodies-upper estimates: the polygonal case and stability. in preparation.

8. S.Y. Cheng, S.T. Yau, On the Regularity of the Solution of the n-dimensional Minkowski Problem. Comm. Pure Appl. Math. 29, 495-516 (1976)

9. D. Ghilli, P. Salani, Stability of isoperimetric type inequalities for some Monge-Ampère functionals. Ann. Mat. Pura Appl. 193(3), 643-661 (2014)

10. H. Groemer, Stability Properties of Geometric Inequalities. Amer. Math. Monthly 97(5) 382$394(1990)$

11. M.N. Ivaki, Centro-affine curvature flows on centrally symmetric convex curves. Trans. Amer. Math. Soc., to appear, arXiv:1205.6456v2.

12. M.N. Ivaki, On the stability of the p-affine isoperimetric inequality. J. Geom. Anal. DOI: 10.1007/s12220-013-9401-1

13. M. Ludwig, M. Reitzner, A classification of $S L(n)$ invariant valuations. Ann. of Math. 172, 1219-1267 (2010)

14. M. Ludwig, General affine surface areas. Adv. in Math. 353, 1767-1779 (2010)

15. E. Lutwak, On some affine isoperimetric inequalities. J. Differential Geom. 23, 1-13 (1986)

16. E. Lutwak, Centroid bodies and dual mixed volumes. Proc. London. Math. Soc. 60, 365-391 (1990)

17. E. Lutwak, The Brunn-Minkowski-Firey theory. II: Affine and geominimal surface areas. Adv. in Math. 118 244-294 (1996)

18. M. Meyer, A. Pajor, On the Blaschke-Santaló inequality. Arch. Math. (Basel) 55 82-93 (1990)

19. C.M. Petty, Affine isoperimetric problems. Ann. N.Y. Acad. Sci. 440, 113-127 (1985)

20. J. Saint-Raymond, Sur le volume des corps convexes symétriques. Séminaire ChoquetInitiation á l'Analyse 1980-81 Exp. No. 11, pp. 1-25. Université Pierre et Marie Curie, Paris, (1981)

21. L.A. Santaló, An affine invariant for convex bodies of $n$-dimensional space. Portugalia Math. 8, 155-161 (1949)

22. R. Schneider, Convex bodies: The Brunn-Minkowski theory, Encyclopedia of Mathematics and its Applications. Cambridge University Press, New York, (2014)

Institut für Diskrete Mathematik und Geometrie, Technische Universität Wien, Wiedner Hauptstr. 8-10, 1040 Wien, Austria

E-mail address: mohammad.ivaki@tuwien.ac.at 